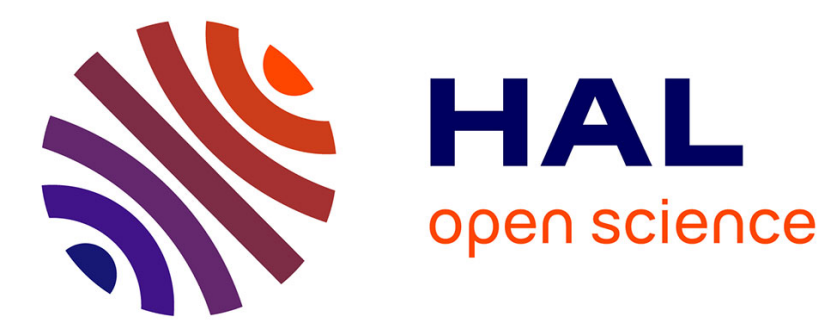

\title{
FUKUSHIMA, UNE DÉMOCRATIE EN SOUFFRANCE
}

\author{
Cécile Asanuma-Brice
}

\section{To cite this version:}

Cécile Asanuma-Brice. FUKUSHIMA, UNE DÉMOCRATIE EN SOUFFRANCE. Outre-terre. Revue européenne de géopolitique, 2012, Haïti, Sichuan, Fukushima États d'urgence, 35-36, pp.457-470. 10.3917/oute.035.0457 . hal-02611177

\section{HAL Id: hal-02611177 https://hal.science/hal-02611177}

Submitted on 17 Jun 2020

HAL is a multi-disciplinary open access archive for the deposit and dissemination of scientific research documents, whether they are published or not. The documents may come from teaching and research institutions in France or abroad, or from public or private research centers.
L'archive ouverte pluridisciplinaire HAL, est destinée au dépôt et à la diffusion de documents scientifiques de niveau recherche, publiés ou non, émanant des établissements d'enseignement et de recherche français ou étrangers, des laboratoires publics ou privés. 


\section{FUKUSHIMA, UNE DÉMOCRATIE EN SOUFFRANCE}

\section{Cécile Asanuma-Brice}

Outre-terre | « Outre-Terre »

2013/1 n³5-36 | pages 457 à 470

ISSN 1636-3671

ISBN 9782358150941

Article disponible en ligne à l'adresse :

https://www.cairn.info/revue-outre-terre1-2013-1-page-457.htm

\section{Pour citer cet article :}

Cécile Asanuma-Brice, "Fukushima, une démocratie en souffrance », Outre-Terre 2013/1 (n 35-36), p. 457-470.

DOI $10.3917 /$ oute.035.0457

Distribution électronique Cairn.info pour Outre-terre.

(C) Outre-terre. Tous droits réservés pour tous pays.

La reproduction ou représentation de cet article, notamment par photocopie, n'est autorisée que dans les limites des conditions générales d'utilisation du site ou, le cas échéant, des conditions générales de la licence souscrite par votre établissement. Toute autre reproduction ou représentation, en tout ou partie, sous quelque forme et de quelque manière que ce soit, est interdite sauf accord préalable et écrit de l'éditeur, en dehors des cas prévus par la législation en vigueur en France. Il est précisé que son stockage dans une base de données est également interdit. 


\section{Fukushima, une démocratie en souffrance}

Cécile Asanuma-Brice ${ }^{1}$

Est-il pertinent de s'interroger sur une poursuite du nucléaire après l'accident de Fukushima?

Le 16 décembre 2012, la 46 élection à la Chambre des représentants a ramené au pouvoir le Parti libéral démocrate de Shinzô Abe, lequel prône une poursuite du programme nucléaire, soit le redémarrage des centrales inactives depuis la catastrophe (seules deux centrales fonctionnant encore aujourd'hui) ainsi que la construction de nouveaux sites. La situation serait donc désormais sous contrôle, ou tendrait à l'être à s'inspirer du thème choisi pour la conférence de l'AIEA (Agence internationale de l'énergie atomique) qui s'est déroulée les 15, 16 et 17 décembre 2012 dans la ville de Koriyama (préfecture de Fukushima) : la sécurité nucléaire.

Car c'est en effet ce qui interroge la population bien au-delà des frontières nippones. Cette question est fondamentale puisqu'elle met en jeu la crédibilité du système mis en place pour assurer la protection humaine. Sait-on gérer un tel accident ? Sommes-nous dans la capacité de protéger les populations où qu'elles se trouvent face aux accidents nucléaires ? La volonté de nos États est-elle de protéger leur population, ou au contraire s'agit-il de défendre d'autres intérêts pour lesquels on serait prêt à mettre en péril une grande partie de ses concitoyens et de leur écosystème ? Et quelle forme cela prend-il ?

C'est un des rôles premiers de l'État, en principe, la protection de sa population, qui est remis en question par cette décision d'une poursuite du nucléaire, et la volonté de continuer à tout prix sur ce chemin prend aujourd'hui des formes traduisant un ébranlement du système démocratique au Japon.

\section{LA REMISE EN CAUSE dU SYSTÈME DÉMOCRATIQUE NIPPON}

Le 25 octobre 2012, lors d'une présentation à Sapporo (Hokkaidô), Matsumura Akio, ancien conseiller de l'ONU vivant aux États-Unis met en garde la population après avoir pris connaissance du contenu de la piscine de combustible à 30 mètres $^{2}$

Chercheur associé au centre de recherche de la Maison Franco-Japonaise.

2 Voir sur le même sujet la déclaration de Matsumura du 3 août 2012, <www.youtube.com/watch?v=0clt0Qfc8UA>. 
au-dessus du bâtiment 4 de la centrale de Fukushima, soit 1535 barres de combustibles nucléaires équivalant à 10 fois la quantité présente lors de l'accident de Chernobyl. L'effondrement de cette piscine pouvant être déclenché par un tremblement de terre de niveau 5 sur l'échelle de Richter revenait à une explosion équivalant à 5005 fois la bombe atomique d'Hiroshima. La réaction en chaîne qui s'ensuivrait rendrait un territoire de $3000 \mathrm{~km}^{2}$ inaccessible à l'homme.

Matsumura déclarant : "Je suis allé rencontrer vos représentants politiques, vos parlementaires, vos élus. Ils ne m'ont pas écouté. La situation est très grave. C'est à vous d'assurer votre protection" .

Le $1^{\text {er }}$ novembre 2012, le détournement du budget de la reconstruction par le gouvernement japonais lui-même est rendu public ${ }^{3}$. Le 6 novembre, le parlementaire Kawada Ryuhei fait une déclaration explosive à la Diète incriminant la spoliation dont la population est victime, d'autant que ces fonds auraient dû être utilisés à la mise en œuvre de la loi « sur la protection des enfants et sur le refuge » votée en juin, qui n'est toujours pas appliquée. Conclusion sans appel : «Un État qui n’est pas en mesure de protéger sa population n'a pas lieu d'exister $»^{4}$

Le 26 novembre 2012, dans une conférence de presse donnée à Tokyo, Mr. Anand Grover, Rapporteur spécial sur le droit de toute personne de jouir du meilleur état de santé physique et mentale susceptible d'être atteint (UN Special Rapporteur on the right of everyone to the enjoyment of the highest attainable standard of physical and mental health), incrimine les autorités pour n'avoir pas distribué des pastilles d'iode aux populations après l'accident et du fait que les mesures du degré de contamination effectuées par le gouvernement étaient faussées ; il met en question l'invitation aux familles par le gouvernement à ne pas procéder à un second examen médical de confirmation pour les enfants qui ont des nodules sur la thyroïde et dénonce les politiques ayant tendu à dissuader les personnes d'opter pour le refuge. Pour déclarer :

" Je demande instamment au gouvernement de veiller à ce que les personnes concernées, en particulier les groupes vulnérables, soient pleinement impliqués dans tous les processus de prise de décision $"^{5}$.

\section{DANS LES FAITS, QUE SE PASSE-T-IL ?}

Une vaste politique incitant la population à oublier l'accident et visant à ce que les populations retournent vivre dans les zones contaminées a été mise en place ; elle

\footnotetext{
3 Cf. Frédéric Charles, "Japon, une partie de l'argent consacré à la reconstruction utilisée pour autre chose ", RFI, $1^{\text {er }}$ novembre 2012.

$4<$ www.youtube.com/watch? $v=z u X d Z I 36 X 7 Q>$.

5 Dans Tokyo Shimbun, 29 novembre 2012, 国連専門家が国・県批判 : ce qui doit inclure leur participation, entre autre, à l'élaboration des enquêtes de santé, à l'attribution des abris d'évacuation et aux opérations de décontamination.
} 
est particulièrement visible dans les mesures pour le relogement des populations, le logement étant un outil efficace de gestion des flux.

Nous avons montré lors de travaux précédents ${ }^{6}$ que ce système de logements publics destinés à une population à faible revenu n'est ni logique, ni naturel. Il est issu, en France, d'un long processus social et politique, combiné à une situation hygiénique déplorable, alors qu'au Japon l'impulsion décisive furent d'abord une catastrophe écologique, le grand tremblement de terre de 1923, puis une catastrophe humaine, la Seconde Guerre mondiale, avec néanmoins instrumentalisation à partir de là à des fins politiques et économiques. Ce sont donc des situations extrêmes auxquelles il a fallu faire face dans l'urgence et qui ont contraint chacune des deux sociétés à penser des solutions nouvelles pour loger les populations. L'État japonais, faute d'avoir su répondre à ses objectifs en termes de rentabilité, allait finalement décider d'interrompre son implication dans le secteur du logement et s'en désengager financièrement à partir de 2005. La triple catastrophe - tremblement de terre, razde-marée, explosion de la centrale nucléaire de Fukushima - qu’a connu le Japon le 11 mars 2011 aurait pu générer un nouvel engagement de l'État dont l'un des rôles est la protection de sa population. Mais la situation actuelle confirme une intention de transférer les prérogatives de l'État-providence au secteur privé. Tout porte à croire que les outils publics originellement mis en place pour assurer la protection de la population sont détournés de leur vocation première pour être utilisés comme outil de contrôle, voire de manipulation de cette même population.

Dans le cas d'une catastrophe nationale comme celle du 11 mars, l'organisme chargé de centraliser les informations et de coordonner la politique en termes d'habitat est le ministère du Territoire, des Infrastructures et des Transports (Kokudôkôtsûshô 国道交通省). À l'aide d'échelles numériques (lesquelles ne sont que des évaluations à un instant $\mathrm{t}$ permettant de donner un ordre de grandeur), les autorités publiques ont mis en place deux volets d'action concernant le relogement des victimes : la mise à disposition gratuite des logements publics vacants à l'échelle du territoire japonais et la construction d'habitats d'urgence.

\section{LES INSUFFISANCES DU SYSTÈME DE LOGEMENTS VACANTS MIS À DISPOSITION DES VICTIMES}

À l'échelle nationale, chaque ville a communiqué à la préfecture dont elle relève un nombre d'habitats municipaux vacants. Les préfectures ont fait de même avec les logements vacants de gestion préfectorale dont elles ont déclaré le nombre total

6 Thèse de doctorat, La transformation de la périphérie urbaine de Tokyo par les organismes de logements publics, septembre 2012, École des hautes études en sciences sociales. 
au ministère du Territoire, des Infrastructures et des Transports. Pour l'ensemble du pays, 24000 logements disponibles (dont 1700 dans la région du Tôhoku) relevant des communes ou des préfectures ont été mis gratuitement à disposition sur une période variant dans un premier temps de 6 mois à un an. Ce délai a été rallongé une première fois jusqu'à la fin mars $2012^{7}$ et devait finalement s'achever le 28 décembre $2012^{8}$. La régie d'État $k \hat{o} d a n^{9}$ a quant à elle offert 5100 logements vacants gratuits pour des durées similaires, dont 130 se trouvent dans le Tôhoku. L'attribution de ces logements se fait par tirage au sort.

Huit mois après la catastrophe, seuls $7900^{10}$ logements communaux et préfectoraux avaient été attribués, soit un tiers du parc; et 940 parmi ceux proposés par la régie kôdan, soit un cinquième. Selon les services d'attribution de l'organisme, ces derniers sont principalement occupés par des familles provenant de Fukushima. Si, deux mois après la catastrophe, on pouvait évoquer la lenteur du système de relogement, le manque d'information quant à ces logements mis à disposition (la plupart des lieux de refuge ne disposant pas d'accès Internet, vecteur essentiel de l'information), l'inquiétude de voir la gratuité des loyers offerts s'interrompre du jour au lendemain, la nécessité d'avoir à retrouver un emploi dans le nouveau lieu de vie et de laisser les biens acquis sur place sans indemnisation permettant un remboursement ne serait-ce partiel des biens abandonnés sont autant de facteurs qui font obstacle à la volonté de refuge exprimée par la population.

Le secteur privé a pallié autant que faire se peut les défaillances du système mis en place par l'administration publique en proposant également des logements gratuits dans tout le pays. Autrement dit, la population s'est mobilisée afin de venir en aide aux victimes. De nombreuses agences immobilières affichaient des propositions de logements ou d'hébergements gratuits, tant en devanture de leur vitrine que sur leur site Internet. Cette procédure était beaucoup plus simple et plus efficace, évitant d'avoir à passer par la longue et lourde procédure d'attribution de logement public.

\section{L'INCITATION AU RETOUR}

La situation a aujourd'hui considérablement évolué. La question du refuge n'est plus liée directement à la catastrophe naturelle du 11 mars, mais elle est imposée par la contamination qui continue de se répandre sur une vaste zone remontant au nord

7 Entretien avec M. Shiotani, section relogement des victimes du tremblement de terre, Urban Renewal (Kôdan), 6 décembre 2011.

8 Cf. "Aide aux réfugiés volontaires dans la préfecture, aide aux enfants et femmes enceintes, le département met à disposition des logements loués dans le secteur privé " (県内自主避難も補助子ども、妊婦世帯支援 県の民間住宅借り 上げ), Fukushima Minpo, 13 novembre 2012.

9 II s'agit d'un organisme mixte créé en 1951, dont le budget est composé de fonds publics et privés (ces derniers occupant une part croissante), et destiné à la création de logements publics et d'aménagements urbains divers.

10 Document du ministère du Territoire, des Infrastructures et des Transports, État de l'emménagement et des logements vacants dans le parc locatif de UR (kôdan), et dans celui des logements municipaux et préfectoraux, 28 novembre 2011. 
de la centrale de Fukushima Daiichi et recouvre une large partie du territoire allant de Fukushima à Tokyo (cf. carte du ministère de l'Enseignement et de la Recherche, décembre 2011).

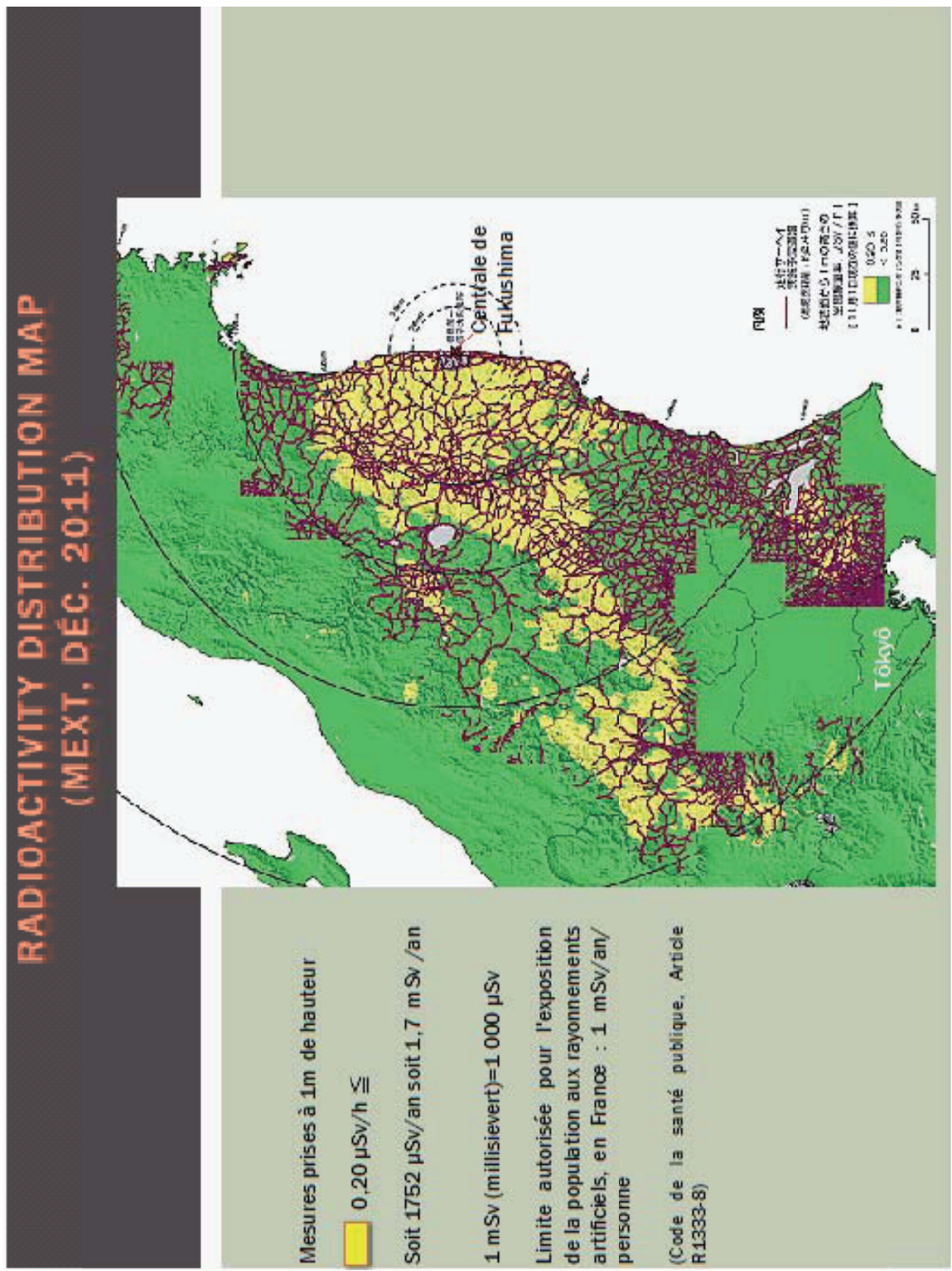


Les personnes réfugiées en conséquence du danger de radioactivité sont désignées comme "réfugiés volontaires ", le gouvernement japonais n’ayant pas reconnu de droit juridique au refuge après les explosions et les fusions de cœur de réacteurs ayant eu lieu à la centrale de Fukushima Daiichi. Une proposition de loi «pour la protection sanitaire des enfants " élaborée en concertation avec diverses associations de citoyens et de juristes regroupés au sein du groupe "Assemblée des citoyens ", shimin kaigi (市民会議) proposant la notion de "droit au refuge " (hinan no kenri) a été votée par la Diète en juin 2012. Mais cette notion reste pour le moment vide de toute implication juridique car elle ne s'accompagne pas de la mise en place d'un système de soutien ou d'indemnisation financière permettant la réalisation concrète du refuge. La politique adoptée tendant au contraire à contraindre les personnes réfugiées au retour dans la préfecture de Fukushima. Ainsi, parmi les mesures de dissuasion apparaissent notamment les points suivants :

- la gratuité du suivi sanitaire n'est assurée que si l'enregistrement de la résidence dans la préfecture de Fukushima est maintenu, ce qui entraîne l'impossibilité de scolariser les enfants dans une autre préfecture ;

- des aides à venir pour le refuge dans la préfecture de Fukushima (appel au retour des populations) vont être mises en place, alors que simultanément la gratuité des logements publics mis à disposition dans 23 préfectures du pays aura pris fin le 28 décembre $2012^{11 .}$

Afin d'inciter la population au retour, une vaste campagne de " nettoyage " des sols a par ailleurs été déclenchée. Selon le rapport du 29 octobre 2012 relatif à la reconstruction de Fukushima, réalisé par les services de la préfecture du même nom, 281 milliards de yens (soit 3 milliards d'euros) allaient être consacrés au nettoyage des sols de la préfecture. Aujourd'hui, l'efficacité de ce nettoyage reste cependant en cause. Le nettoyage consiste à passer les maisons au jet d'eau (les particules retombant au sol pour s'y infiltrer), à élaguer le feuillage des arbres et à racler quelques centimètres de terre en surface. Si le taux de radioactivité baisse effectivement au moment du nettoyage, il remonte à la première pluie. Le nettoyage d'une habitation revient en moyenne à 500000 yens (4 720 euros). Autant dire que c'est aujourd'hui un marché fabuleux qui s'est ouvert pour la main-d'œuvre du bâtiment, une aubaine qui permet enfin de sortir de la crise de la construction laquelle sévissait depuis 2007. Les ouvriers affluent de toute part.

Les habitants que nous avons rencontrés ne semblent pas dupes. "Le nettoyage n'existe pas ", "le nettoyage est impossible ", "qu'ils nous donnent l'argent dépensé en vain pour le nettoyage afin de financer des indemnités de déménagement ! , nombreux sont les témoignages de ce genre que nous avons pu entendre. Le simple bon

11 " Aide aux réfugiés volontaires dans la préfecture, aide aux enfants et femmes enceintes, op. cit. 
sens suffit à chacun pour comprendre que les particules s'imprègnent dans le sol et se déplacent d'un voisin chez l'autre. L'illusion d'une efficacité du nettoyage des sols est toutefois entretenue et participe en fait d'un lavage des cerveaux dont l'objectif principal est de rassurer et de procurer aux populations un sentiment de sécurité et de protection qu'elles n'ont à l'évidence absolument pas. Ainsi, le maire d'Iitate (à 40 kilomètres de la centrale), une des villes qui enregistre les taux les plus élevés de contamination, a-t-il déclaré à sa population qu'elle pourrait revenir y habiter d'ici 5 ans.

Parmi les autres mesures visant à promouvoir la « renaissance » du centre urbain de la ville de Fukushima sont organisés tous les week-ends des événements culturels divers sur la place du centre ville : "Voilà six mois que nous n'avons plus un weekend de libre", expliquent les animateurs culturels de la ville ${ }^{12}$.

Malgré ces mesures incitatives, voire coercitives, le nombre de réfugiés volontaires semble à en croire les associations citoyennes de gestion des réfugiés en croissance permanente. Plus de 3000 réfugiés dans la préfecture d'Hokkaidô ${ }^{13}$, plus de 13000 dans la préfecture de Yamagata ${ }^{14}$, au total 63960 personnes qui seraient réfugiées hors de la préfecture de Fukushima, alors que 100000 le seraient dans les zones moins contaminées à l'intérieur de cette préfecture. Les associations s'élèvent contre les déclarations des autorités publiques selon lesquelles le nombre de personnes réfugiées serait en diminution. Il s'agit en effet ici avant tout d'induire un élan de retour, de rassurer les personnes encore sceptiques en leur montrant que les « autres » commencent à rentrer et qu'elles feraient bien d'en faire autant.

2 Entretien réalisé au cinéma de Fukushima, Forum Fukushima, le 29 octobre 2012.

13 Selon les données de la préfecture de Hokkaidô en novembre 2012.

14 Résultat de l'enquête du 16 novembre 2012, préfecture de Yamagata，避難者アンケート調查集計結果について，山形 県広域支援対策本部避難者支援班). 
Nombre de réfugiés à l'intérieur et à l'extérieur de la préfecture de Fukushima

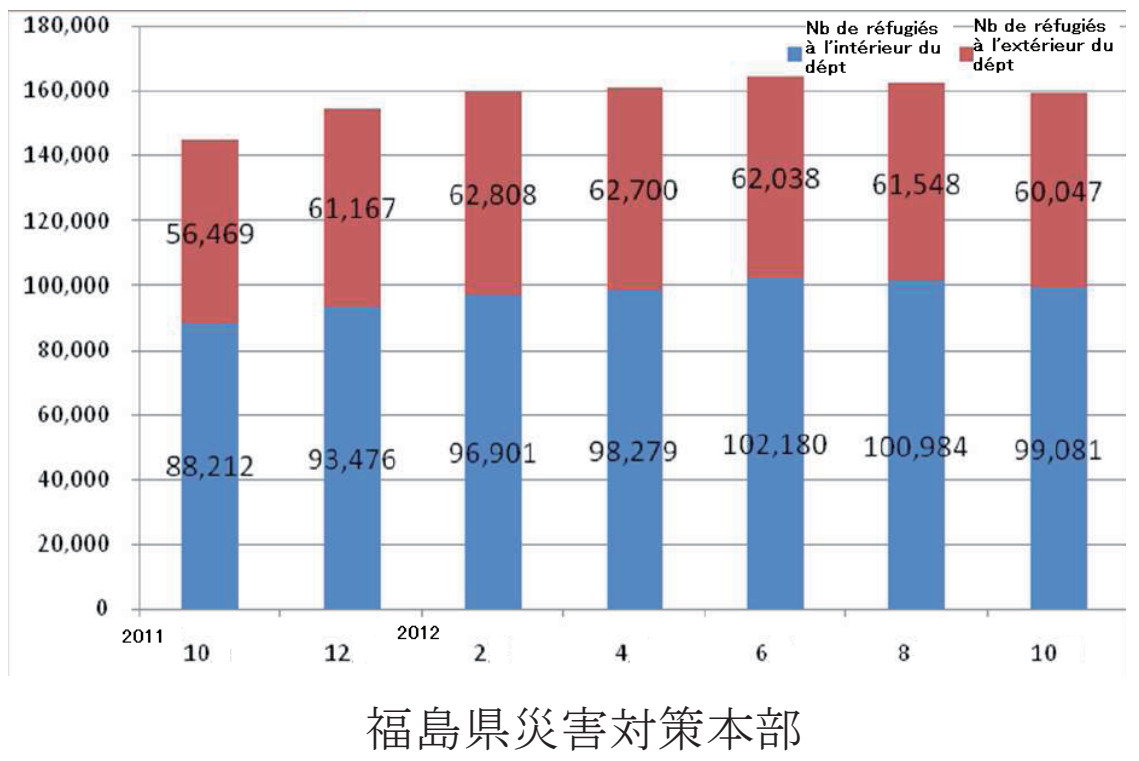

Rapport du 29 octobre 2012, préfecture de Fukushima, service de gestion en cas de désastre

Carte de la répartition de la population réfugiée hars du département de Fukushima (63 959 pers.)

\begin{tabular}{|c|c|}
\hline Préfectures & Population \\
\hline Yamagata & 13033 \\
\hline Tôkyô & 7570 \\
\hline Niigata & 6683 \\
\hline Sałama & 4593 \\
\hline Ibaraki & 3612 \\
\hline Chiba & 3214 \\
\hline Tochigi & 2710 \\
\hline Kanagawa & 2572 \\
\hline Gunma & 1921 \\
\hline Hokkaidô & 3000 \\
\hline Miyagi & 1810 \\
\hline Akita & 1174 \\
\hline $\begin{array}{l}34 \text { préfectures } \\
\text { (divers) }\end{array}$ & 12070 \\
\hline Total & 63959 \\
\hline
\end{tabular}

Sources Prefecture de Fuaushima, 260101/2012

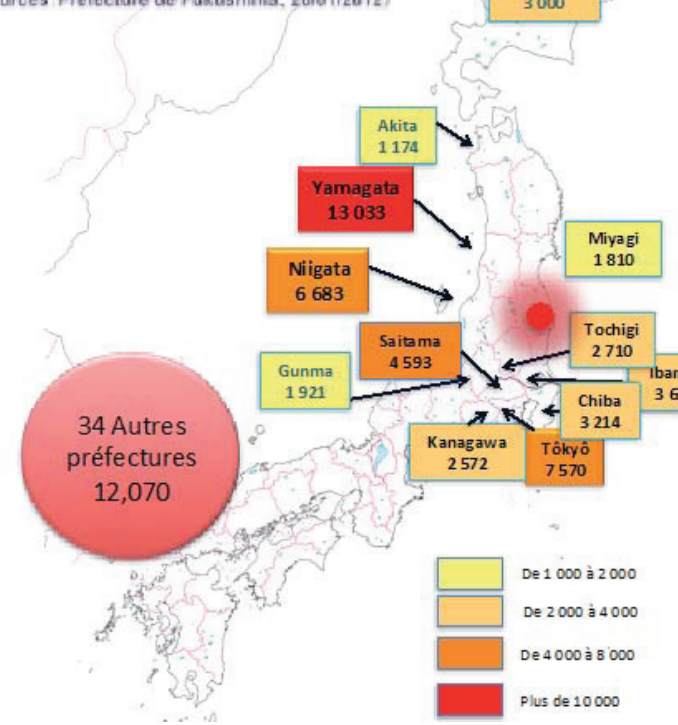


Le nombre de réfugiés déclaré par les autorités publiques, qui est la seule source dont nous disposons actuellement, reste largement sous-estimé si l'on en croit l'Association des réfugiés volontaires. ${ }^{15}$ Cette Association regroupe 35 ménages réfugiés dans la préfecture de Yamagata. Tous habitaient la ville de Fukushima. Ils réclament le droit au suivi sanitaire, au refuge et à des indemnités leur permettant de pouvoir se reloger. Les représentants de cette Association ont cherché à comprendre la façon dont les statistiques sur le nombre de réfugiés étaient élaborées. Les réfugiés doivent d'eux-mêmes se rendre dans les bureaux de la préfecture et s'inscrire dans le "système d'information concernant les réfugiés " (Hinansha jôhô shisutemu). Ce système étant inconnu de la population, les inscriptions sont très peu nombreuses. Selon les propos des représentants de l'Association, aucun des 35 ménages membres n'y est enregistré à ce jour.

Suite à ces protestations, les chiffres des réfugiés volontaires ont été revus à la hausse par l'Agence de la reconstruction dans un rapport daté du 12 décembre $2012^{16}: 321000$ personnes seraient réfugiées, dont 98300 personnes à l'intérieur de la préfecture de Fukushima ; les préfectures alentour sont celles qui comptent le plus de réfugiés à l'heure actuelle, soit Miyagi (112 000 personnes), Iwate (41 626 personnes) et Yamagata (10 693 personnes).

\section{LOGEMENTS PROVISOIRES ET ISOLEMENT DE LONGUE DURÉE}

De même que pour la disponibilité en logements publics, chaque commune dépose sa demande de logements provisoires à la préfecture laquelle centralise les documents à son échelle pour relayer l'information auprès du ministère de la Santé et du Travail (Kôseirôdôshô 厚生労働省) ${ }^{17}$. Ces logements sont financés par la « caisse spéciale d'aide en cas de cataclysme ». Les données sont ensuite fournies au ministère $\mathrm{du}$ Territoire, des Infrastructures et des Transports qui fait appel à des promoteurs privés pour la construction. Les travaux peuvent être gérés selon les cas par la régie kôdan. 

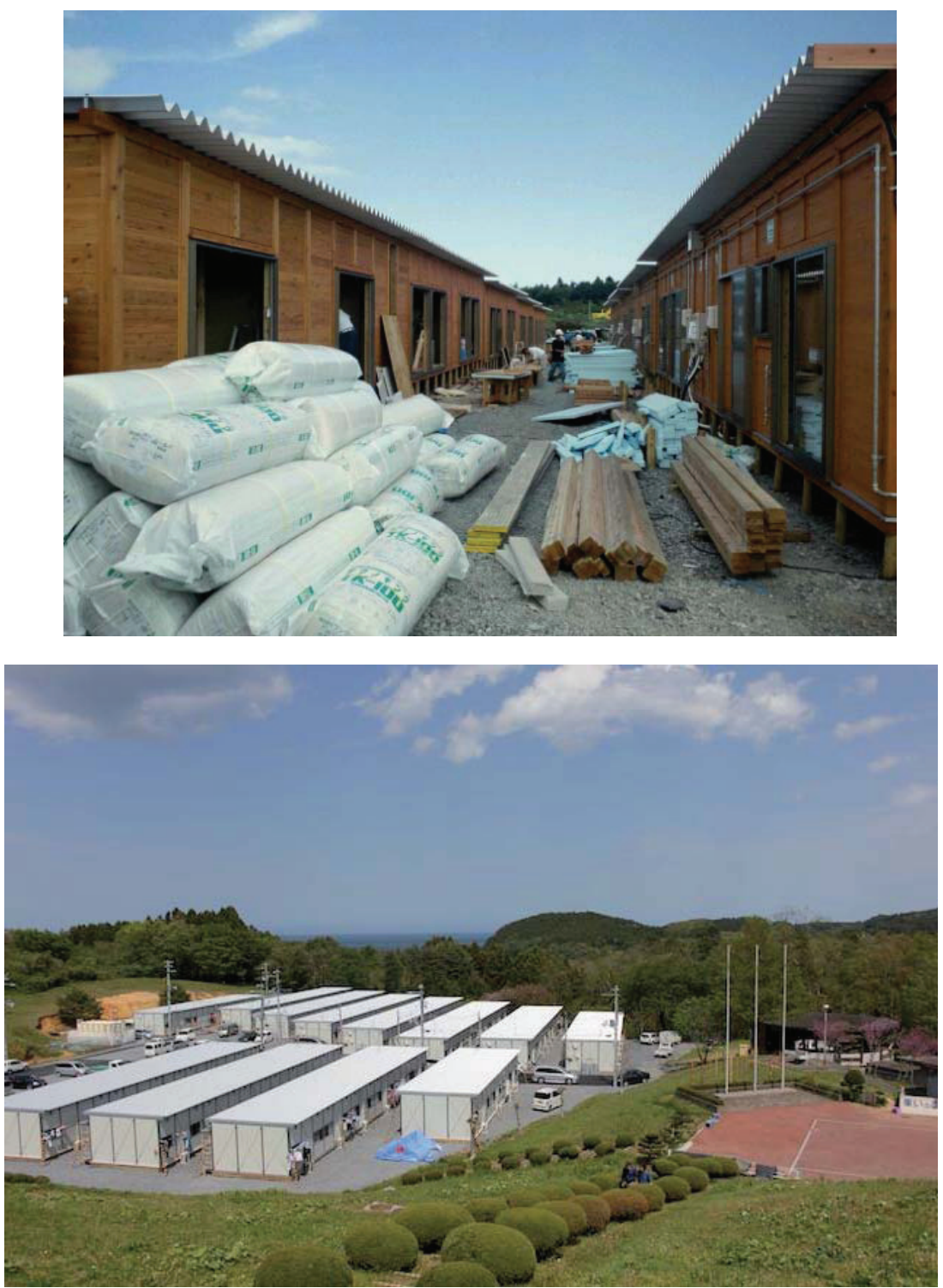

Les logements provisoires de Minamisôma et Aizuwakamatsu, novembre 2012

Le nombre de logements provisoires d'urgence requis pour permettre le relogement des populations sinistrées dans leur ensemble était de 52743 unités selon les données établies au 28 novembre 2011 par le ministère du Territoire, des Infrastruc- 
tures et des Transports. La quasi-totalité de ces habitations a été réalisée, puisque l'on en comptait 52041 selon les données nationales en décembre $2011^{18}$.

La grande majorité d'entre eux se trouve dans la préfecture de Miyagi (22 095), principalement touchée par le raz-de-marée. 14000 unités ont été construites à Iwate et environ 16775 dans la préfecture de Fukushima. Les premiers d'entre ces logements ont été établis dans cette dernière, sur des zones que nous savons aujourd'hui contaminées. Certaines familles se retrouvent captives de ces habitations à surface réduite, puisqu'elles ne disposent que de $19,8 \mathrm{~m}^{2}$ pour une personne seule à 39,6 $\mathrm{m}^{2}$ pour une famille de cinq personnes. Limitant leur sortie à l'extérieur au strict minimum, ces personnes sont aujourd'hui à peu près prisonnières de leur habitat et se trouvent parfois dans une situation d'isolement préoccupante.

Logements provisoires d'urgence et contamination radioactive : une répartition géographique similaire

Source : Carte réalisée par Cécile Asanuma-Brice (situation en février 2012) à partir de données de la préfecture de Fukushima

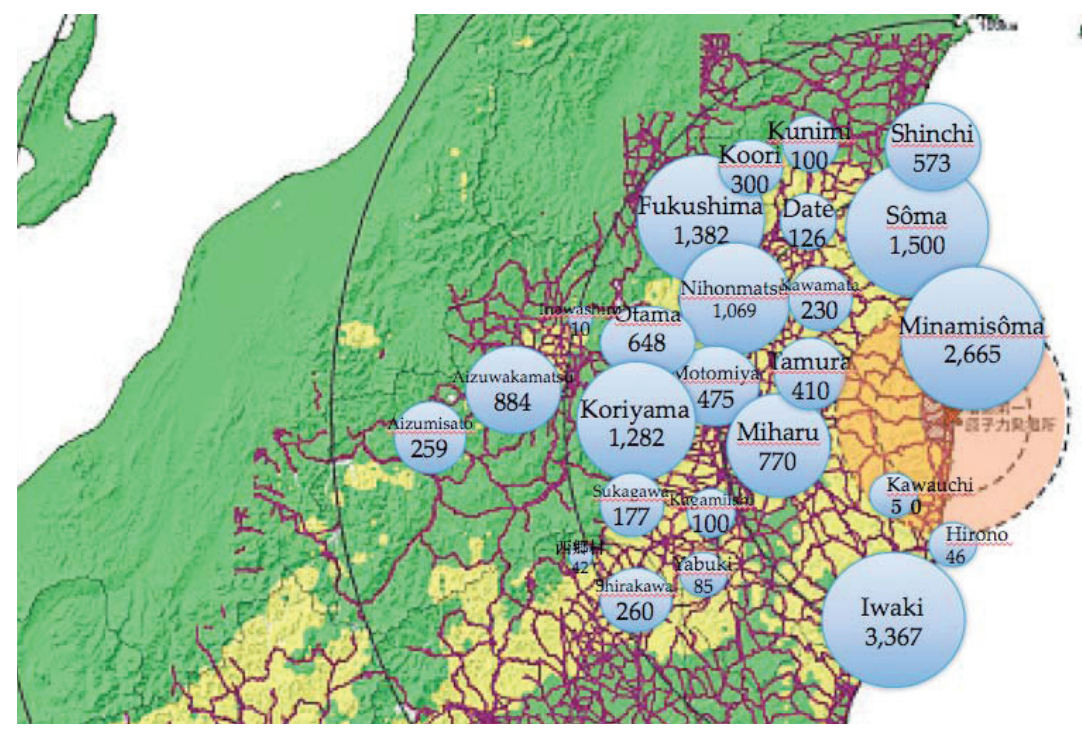

Cette carte met en évidence la localisation des logements provisoires publics (16 810 unités) sur les zones contaminées. La logique de rentabilisation économique, qui est devenue le seul et ultime moteur de l'aménagement urbain, ne semble pas être remise en cause et conduit le gouvernement non plus à protéger sa population, mais à la placer en situation de péril

18 Kokudôkôtsûshô, ministère du Territoire, des Infrastructures et des Transports, décembre 2011. 
Le problème de l'isolement n'est toutefois pas limité à la région de Fukushima et à la récente catastrophe. Le séisme de Kobe avait en son temps révélé la question de la mort par isolement (kodokushi孤独死) dans les logements provisoires et les problèmes d'alcoolisme afférents ${ }^{19}$.

Ce problème est également présent, depuis plusieurs années maintenant, au sein des cités de logements publics en périphérie de Tokyo. Selon une enquête menée par le ministère de l'Intérieur (naikakuhu内閣府), 131 suicides ont eu lieu entre mars et juin dans les trois préfectures d'Iwate, Miyagi et Fukushima ${ }^{20}$. De juin à septembre 2011, dix cas de suicides ont été recensés dans les logements provisoires du seul département d'Iwate ${ }^{21}$ : six hommes et quatre femmes, dont l'âge s'échelonne entre trente et quatre-vingts ans. Les causes sont multiples, mais au désespoir d'avoir tout perdu vient s'ajouter l'isolement des logements provisoires, parfois implantés dans des rizières éloignées de tout. Celui-ci est tel qu'un corps a été retrouvé une semaine après le décès ${ }^{22}$.

Nos visites dans le cités de logements provisoiresn de Minamisôma et d'Aizuwakamatsu nous ont permis de mettre en évidence la situation des résidents de ces cités. Les personnes que nous avons rencontrées et interviewées logeaient toutes originellement dans les villages voisins de la centrale (Ôkuma, Futaba, Naraha, Tomioka). Elles ont accès à leur habitation d'origine sur obtention d'une autorisation de la Préfecture, et ce deux heures par mois. Au moment de leur relogement, TePCo (Tokyo Electric Power Company) leur a versé 1 million de yens (9 500 euros) de dédommagement. Cette même entreprise leur a ensuite demandé de rembourser cet argent, afin qu'elle puisse en calculer une répartition entre tous les habitants sous forme d'un salaire mensuel de 100000 yens /personne/mois (942 euros). Ces personnes vivent maintenant avec ce seul revenu, dans des logements moins provisoires qu'il n'y paraît, en suspens. Les plus âgées d'entre elles (86 ans et plus) disent ne pas vouloir rentrer, quant bien même elles le pourraient. Les plus jeunes disent vouloir partir, refaire leur vie ailleurs. "Que ferons-nous s'il n'y a plus de jeunes au village?" " clament les personnes âgées qui se trouvent dans ces logements et restent donc là, âmes en peine mais heureuses pour certaines d'être avec les leurs, sans attente d'un lendemain : " Nous avons le sentiment de courir un marathon pour lequel il n'y a pas de but. Et nous courons, nous courons... $»^{23}$.

19 Cf. Ogino Masahiro, Fissures : Kobe, 17 janvier 1995, le séisme, Grenoble, Éditions de la Villette, 1998.

20 《 6 月の震災 3 県自殺 震災関連が 1 割 内閣府調査》 (La catastrophe de juin - suicides dans les trois préfectures : $10 \%$ en rapport avec le séisme, enquête du ministère de l'Intérieur ), Sankei News, 5 août 2011.

21 《岩手県が自殺対策プラン対策へ 震災関連は 10 人》 (Vers la mise en place d'un plan de lutte contre le suicide dans le département d'Iwate 10 suicides en lien avec la catastrophe), Nishi Nihon Shimbun, 21 novembre 2011.

22 《仮設住宅で 60 歳男性が自殺か…宮城・石巻》(Le suicide d'un homme de 60 ans dans un logement provisoire... Miyagi, Ishinomaki), Yomiuri Shimbun, 16 septembre 2011.

23 Entretien dans la cité de logements provisoires de Minamisôma, 3 novembre 2012. 


\section{RECONSTRUIRE EN ZONE CONTAMINÉE}

Reconstruire en zone contaminée ne semble plus poser de problème. Dans un document daté du 29 octobre $2012^{24}$, la préfecture de Fukushima, après avoir indiqué les taux de contamination des diverses communes de la région (cf. infra), expose l'avancement de son plan de reconstruction.

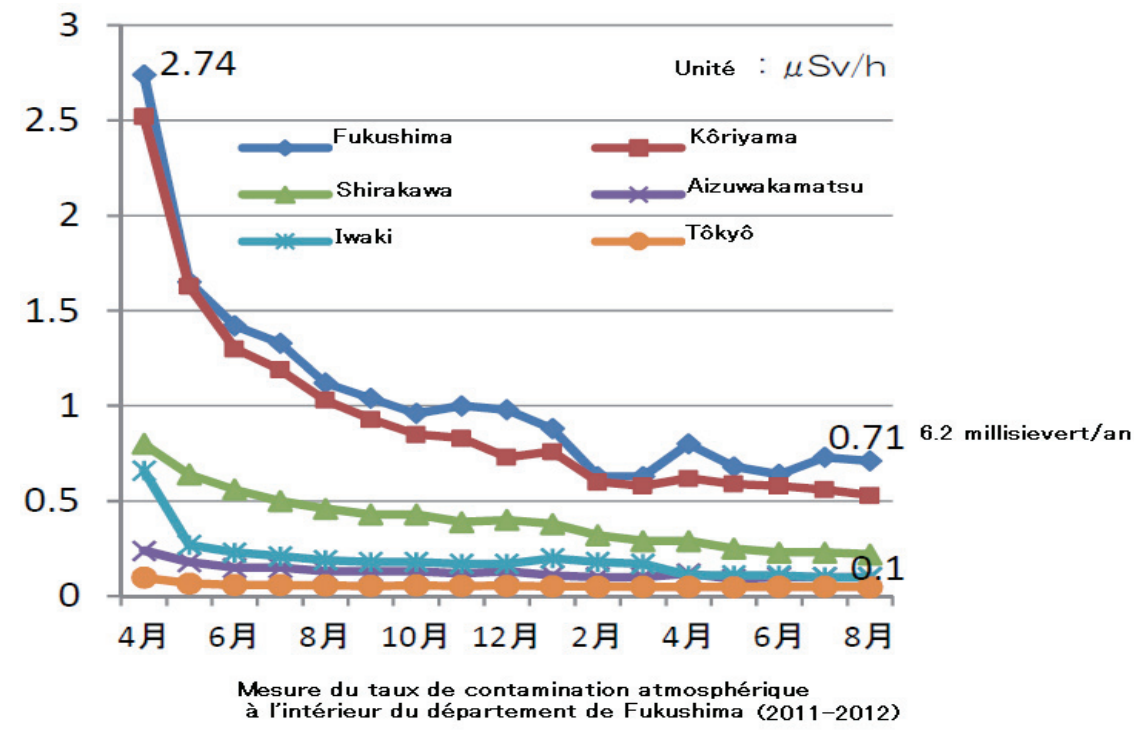

Mesures effectuées par le centre de sécurité sanitaire de Tôkyô (Shinjuku)

Les documents officiels utilisent comme unité de valeur les $\mu \mathrm{Sv} / \mathrm{h}$ (microsievert/ heure) pour l'expression des taux de contamination. Ainsi apparaissent des chiffres de faible valeur, alors que les normes internationales sont exprimées en mSv/an (millisievert/an). Le document ci-dessus affiche un taux de $0,71 \mu \mathrm{Sv} / \mathrm{h}$ à Fukushima. Cela signifie que le taux moyen dans la ville de Fukushima est aujourd'hui de 6,2 $\mathrm{mSv} / \mathrm{an}$, soit plus de six fois la norme internationale, et ce sans prendre en considération des zones comme le quartier de Watari (quartier central administratif et résidentiel de Fukushima) où la contamination sur certains points chauds peut atteindre entre 20 et 200 millisievert/an.

Les courbes de contamination sont exposées dans le document official supra parallèlement à un tableau de l'état d'avancement des travaux de reconstruction. Autrement dit, le document nous indique que la préfecture a largement entamé, voire parfois à peu près achevé (à 91,1\%) pour ce qui est des équipements publics,

24 Journal de la reconstruction de Fukushima (Fukushima fukkô no ayumı), préfecture de Fukushima, service de gestion en cas de désastre, 20 octobre 2012. 
son plan de reconstruction. Ainsi, sur les 81092 unités de logements dont elle avait programmé la construction, 34828 unités soit $42,9 \%$ sont prêtes à l'usage.

La préfecture de Fukushima prévoit également de relancer le tourisme dans la région, avec notamment la réouverture du centre de loisirs aquatique Aquamarine Fukushima.

L'audit commandité par le gouvernement lui-même donnait le résultat suivant : la moitié du budget de 144 milliards d'euros a été détourné pour des investissements sans aucun rapport avec la reconstruction. Et ce alors que plus de 300000 habitants séjournent toujours dans les logements provisoires. 